\title{
Improved Tracking Using a Hybrid Optcial-Haptic Three-Dimensional Tracking System
}

\author{
${ }^{1,2} \mathrm{M}$ 'hamed Frad,${ }^{1}$ Hichem Maaref, ${ }^{1}$ Samir Otmane, ${ }^{2}$ Abdellatif Mtibaa \\ ${ }^{1}$ IBISC Laboratory, Université d'Evry Val d'Essonne, France \\ ${ }^{2} \mathrm{E} \mu \mathrm{E}$ Laboratory, University of Monastir, Tunisia
}

\begin{abstract}
The aim of this paper is to asses to what extent an optical tracking system (OTS) used for position tracking in virtual reality can be improved by combining it with a human scale haptic device named Scalable-SPIDAR. The main advantage of the Scalable-SPIDAR haptic device is the fact it is unobtrusive and not dependent of free line-of-sight. Unfortunately, the accuracy of the Scalable-SPIDAR is affected by bad-tailored mechanical design. We explore to what extent the influence of these inaccuracies can be compensated by collecting precise information on the nonlinear error by using the OTS and applying support vector regression (SVR) for calibrating the haptic device reports. After calibration of the Scalable-SPIDAR we have found that the average error in position readings reduced from to $263.7240 \pm 75.6207 \mathrm{~mm}$ to $12.6045 \pm 8.4169 \mathrm{~mm}$. These results encourage the development of a hybrid hapticoptical system for virtual reality applications where the haptic device acts as an auxiliary source of position information for the optical tracker.
\end{abstract}

Keywords-virtual reality; Scalable-SPIDAR; support vector regression; hybrid tracking system

\section{INTRODUCTION}

Optical trackers provide a reliable and accurate position tracking for virtual reality applications. The optical tracking relies on measurements of reflected or emitted light [1]. It is therefore, evident, that there must be a clear free line-of-sight between the light source and camera assembly. This requirements turns out to be difficult to maintain at all times and a partial occlusion may turn out to be the biggest problem as it results in a tracking-loss.

Scalable SPIDAR haptic device is not dependent on free line of sight, is suitable for a large-scale immersion and is significantly cheaper than commercial optical trackers. This device was used in virtual reality to track and measure motion of user's hand as well as to enable large scale immersion. Unfortunately, previous experiments with Scalable-SPIDAR [2] have revealed significant inaccuracies between caused by design structure shortcomings.

Our work focus on combining a human scale haptic device and an optical tracker in hybrid tracking system; this approach aims to overcome obstructions of line-of-sight while maintaining an interrupted and accurate tracking for applications in virtual reality.

This paper deals with an important point in the feasibility of such a system. For efficient application of a hybrid tracking system sufficient registration accuracy between the two components has to be achievable. This essential since the
Scalable-SPDIAR is intended to serve as secondary source of position information for the optical tracker and therefore have to be reported in the coordinate system of the optical tracker.

Furthermore, it has to be assessed to what extent systematic errors in the Scalable-SPIDAR reading positions that stem from the bad-tailored mechanical design can be calibrated.

\section{PREVIOUS WORK}

In the last decade several hybrid tracking systems have been proposed in the literature. The suggested methods attempt to compensate for the shortcomings of each tracking technology by using multiple measurements to provide robust tracking. State et al's [3] work developed a hybrid tracking scheme that has the registration accuracy of vision-based tracking systems and the robustness of magnetic tracking systems. Similar to this, in [4] the authors built a hybrid tracking system integrating optical and magnetic tracking. The built system is faster than a standalone optical tracker and outperforms a magnetic system in term of accuracy.

You et al [5] presented a hybrid approach with integrated inertial and vision tracking technologies. They use the complementary nature of these two tracking technologies to overcome the shortcomings in each separate component. In [6], an image-based system is coupled with an inertial in order to provide robust and accurate tracking. In fact, in cases when the image based system fails due to abrupt movements, the inertial system takes over.

Birkfellner et al [7] developed a hybrid tracking system that combines an electromagnetic tracking system and optical tracker in order to avoid obstructions of the line-of-sight necessary for the operation of the OTS while maintaining an interrupted tracking and the accuracy needed in computer aided-surgery. More recently Harders et al [8] introduced a hybrid tracking method that combines the IR optical tracker with a vision based tracking approach

\section{VIRTUAL REALITY SETUP}

For our experiment, we used an infrared 6DOF optical tracking system (ARTrack1/Dtrack) with an accuracy from 0.4 to $1.4 \mathrm{~mm}$. The infrared (IR) cameras ARTrack1 illuminate the measurement volume by an IR flash. They are able to recognize retro reflective markers and they compute the marker positions in image coordinate (2D) with high precision [9]. 
The IR optical tracker is combined with a human scale haptic device called Scalable-SPIDAR [10] for Space Interface Device for Artificial Reality. The device is derived from the original desktop SPIDAR which was developed by Hirata and Sato [11]. The scalable-SPIDAR is composed of a cubic frame that encloses a cave-like space, where the use can move around to perform large scale movements. The front side of the device holds a large screen where a generated virtual world is displayed. The device has 8 couples of DC motor/rotary encoder mounted in the corners of the cubic frame. Position of the user's hands can be measured by the length of the strings. The length of a string is known by reading the values from the rotary encoder. The Scalable-SPIDAR haptic device is however is subject to inaccuracies due shortcomings in the mechanical structure design.

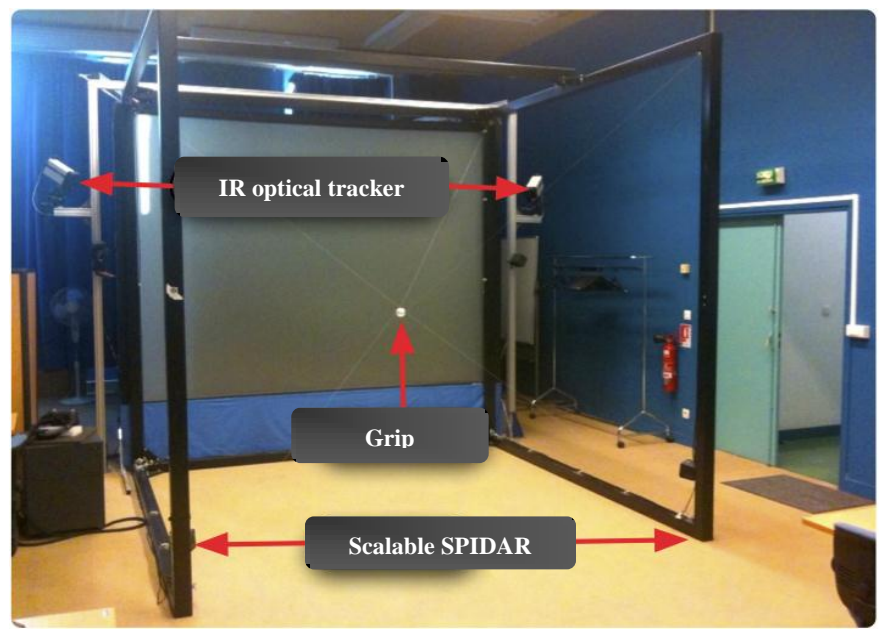

Fig. 1. View depicting all the components of our VR setup. The IR optical tracker (ARTrack1) was mounted below the Scalable-SPIDAR haptic device

\section{METHODS}

In order to access the achievable accuracy from this hybrid tracking setup, we had to perform the following steps:

- Register the coordinate systems $\operatorname{Ref}_{\text {Haptic }}$ and Ref $\mathrm{Rptical}$ in order to merge the data from the different systems modalities such that the position data from the Scalable SPIDAR are reported in $\operatorname{Ref}_{\text {Optical }}$.

- Compensate erroneous position readings from the Scalable-SPIDAR caused by bad-tailored design.

\section{A. Registration of tracking systems}

The IR optical tracker determines the coordinates of retro reflective markers within the measurement volume. In order to acquire 3D point measurements of the haptic point in the world (i.e. Optical tracker) coordinate system an IR marker is attached at the end of the Scalable-SPIDAR grip.

A custom procedure allows the user to accomplish the Scalable-SPIDAR initialization in precise and repeated manner. Using the IR optical tracker, the user can accurately place to fixed known location within the working volume: the Scalable-SPIDAR origin.

Following his, we are able to report the same location in a common world coordinate system. Let $\mathrm{p}_{\mathrm{i}}^{\text {RefOptical }}$ and $\mathrm{p}_{\mathrm{i}}^{\text {RefHaptic }}$ represent the position of the grip, to which attached an IR marker, respectively in the haptic and optical coordinate systems. The position of the grip is determined by: $\overrightarrow{\mathrm{p}}_{\text {RefOptical }}=\overrightarrow{\mathrm{p}}_{\text {RefHaptic }}+\overrightarrow{\mathrm{t}}$ where $\overrightarrow{\mathrm{t}}$ is the translation vector between the Optical Tracker and the Scalable-SPIDAR origins.

\section{B. Calibration of the Scalable-SPIDAR haptic device}

Scalable-SPIDAR is a multi-modal haptic device for large scale virtual environment. It provides a workspace that is large enough to cover almost the measurement volume seen by the infrared cameras. However, due to various limitations, it is subject to inaccuracies and therefore cannot provide faithful data rendering. Problems are mainly caused by shortcomings in mechanical structure design. The calibration method involves several steps: characterizing the Scalable-SPIDAR haptic device and then applying methods to correct errors in the reported position. With our setup, we calibrated the Scalable-SPIDAR for a working volume of $1 \mathrm{~m} \times 1 \mathrm{~m} \times 1 \mathrm{~m}$.

\section{1) Characterization Protocol}

In order to characterize the Scalable-SPIDAR haptic device, we collect tuples that consist of the tracked data and the "truth values" what a convenient reference should an accurate reference report. The accuracy of these truth values is crucial of the calibration method. Therefore, we use the IR optical tracker available within our setup. To provide for welldistributed data that can be collected, a volumetric calibration protocol is proposed. To this end, the screen of our setup is filled by a virtual grid divided into a sequence of a small cubes. Each small cube corresponds to a sub-space of the Scalable-SPIDAR workspace.

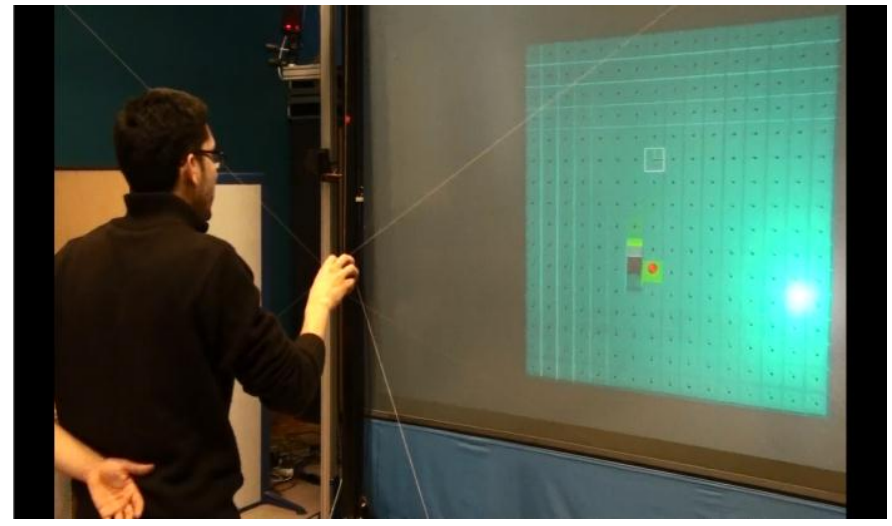

Fig. 2. Display in the Scalable-SPIDAR of boxes to collect. The virtual grip (the red sphere) is moved inside colored box to collect point

A user holds the device's grip s reasonably straight and moves it until the virtual grip ranges inside the cube. Programs record then the position given by the IR optical tracker and the haptic device. Once these positions recorded, the cube vanishes ensuring that only one measurement was associated to this sub-space. In practice a volume of $1 \mathrm{~m}^{3}$ is considered. This volume is divided into 4096 small cubes. Hence 4096 point measurements are sampled inside the virtual grid. The proposed protocol sounds well for gathering a large number of data points with pretermitted distribution using a quasi-static collection mode. 
At each of the resulting 4096 points, measurements reported by the two devices were taken, and the position error was determined as the distance between the tracked position by the Scalable-SPIDAR and the corresponding reference position as reported by the optical tracking system (OTS):

$$
\mathrm{err}_{\mathrm{pos}}=\sqrt{\left(\mathrm{x}_{\mathrm{OTS}}-\mathrm{x}_{\mathrm{S} \_ \text {SPIDAR }}\right)^{2}+\left(\mathrm{y}_{\mathrm{OTS}}-\mathrm{y}_{\mathrm{S} \_ \text {SPIDAR }}\right)^{2}+\left(\mathrm{z}_{\mathrm{OTS}}-\mathrm{z}_{\mathrm{S} \_ \text {SPIDAR }}\right)^{2}}
$$

Where $\left(x_{S_{-} S P I D A R}, y_{S_{-} S P I D A R}, z_{S_{-} S P I D A R}\right)$ is the position reported by the Scalable-SPIDAR and $\left(x_{\text {OTS }}, y_{\text {OTS }}, z_{\text {OTS }}\right)$ is the reference position.

Fig 3 represents the position errors spatially at each reference position $\left(x_{\text {OTS }}, y_{\text {OTS }}, z_{\text {OTS }}\right)$, with the error magnitudes proportional to the corresponding circle diameters. The plot clearly shows that errors are more pronounced when the grip is manipulated away from the center of the workspace toward the edges of the cubic frame.

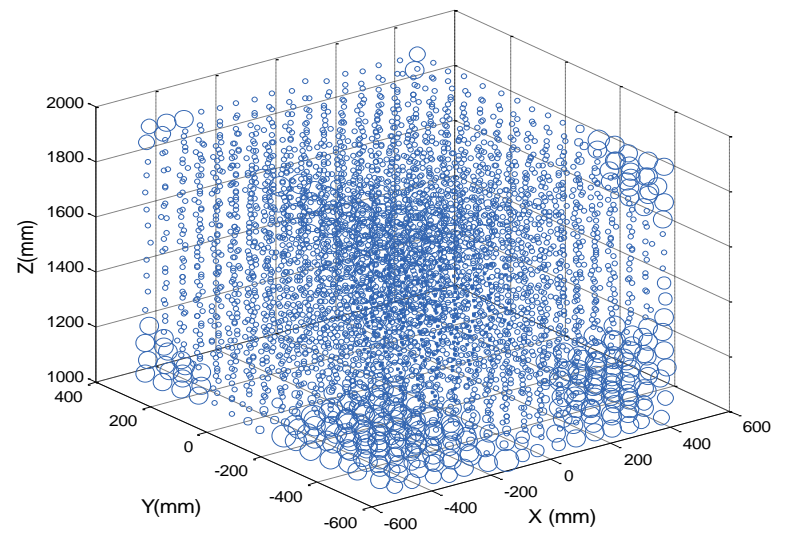

Fig. 3. Distance errors represented spatially

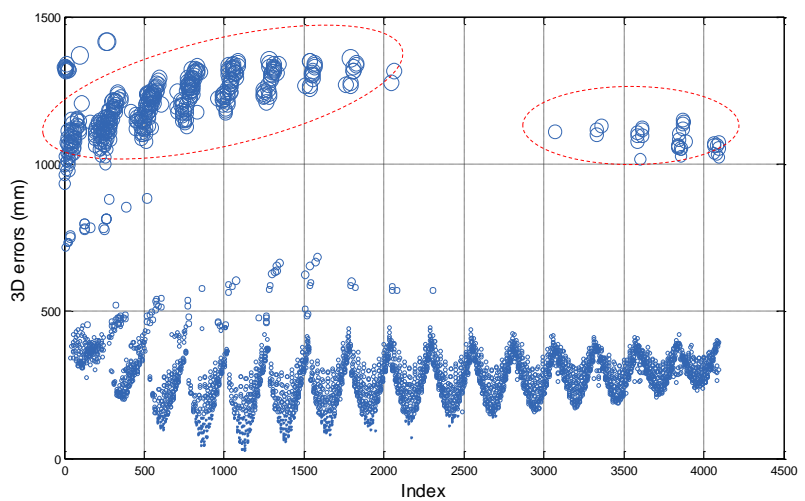

Fig. 4. Distance errors represented in Sequence

In figure 4 , position errors are plotted as a function of the sequence in which they were collected from the front of the volume to the back. Plotting distance errors as 1D plot results in the loss of a lot of spatial information, but still shows the same trend, and from the plot's periodicity we can infer that errors increase at the volume edges. Note the clusters of large error in the upper left and right corner of plot, these groups of outliers are removed using boxplot method.

2) Calibration technique: Support Vector regression $(S V R)$

A function approximation problem can be expressed as to find $a$ function $\mathrm{f}$ from $\mathrm{a}$ set of observations, $X=\left\{\left(\overrightarrow{x_{1}}, y_{1}\right),\left(\overrightarrow{x_{2}}, y_{2}\right), \ldots .,\left(\overrightarrow{x_{N}}, y_{N}\right)\right\}$ with $\overrightarrow{x_{i}} \in R^{m}$ and $y_{i} \in R$ where $\mathrm{N}$ is the number of training data, $\overrightarrow{x_{i}}$ is the $i$ input vector, and $y_{i}$ is the desired output for the input $\vec{x}_{i}$. Based on the support vector machine theory, SVR is to approximate the given observations in an $m$-dimensional space by a linear function in another feature space. The function in SVR is determined as [12]:

$$
f(\vec{x}, \vec{\theta})=\langle\vec{\theta} \cdot \Phi(\vec{x})\rangle+b
$$

Where $\langle.,$.$\rangle is an inner product defined on F, \Phi($.$) is$ nonlinear mapping function from $R^{m}$ to $F, \vec{\theta} \in F$ is a weight vector to be identified in the function, and $b$ is a threshold. Generally, the considered cost function is [31] [32]:

$$
R_{S V}[f]=R_{e m p}[f]+C \cdot\|\vec{\theta}\|^{2}
$$

where

$$
R_{e m p}[f]=\frac{1}{N} \sum_{i=1}^{N} L\left(y_{i}-f\left(\overrightarrow{x_{i}}, \vec{\theta}\right)\right)=\frac{1}{N} \sum_{i=1}^{N} L\left(e_{i}\right)
$$

[13], [14], $L\left(y_{i}-f\left(\overrightarrow{x_{i}}, \vec{\theta}\right)\right)$ is the loss function measuring the error between $\mathrm{y}$ and the estimated output $f\left(\overrightarrow{x_{i}}, \vec{\theta}\right)$ for a given $\vec{x}$, and $C \succ 0$ is a regular constant. The goal of adding the regularization term is to maintain the weight vector as small as possible in the approximation process. When over fitting phenomena happens, some undesirable information, has been modeled in the function. Those undesirable signals usually are not smooth, and as result, some parameters may become large to accommodate such behaviors. Therefore, in (1.3) the cost function has incorporated the intention to minimize $\vec{\theta}$, which in turn, reduces the model complexity. In other terms, the regularization term in (1.3) controls the tradeoff between the approximation accuracy and the model complexity in order to provide good generalization performance accuracy [15].

In classic SVR, the $\varepsilon$ - insensitive function is used as the loss function (1.3). It was first presented in the original SV algorithm [16], [17]. The $\varepsilon$ - insensitive function is defined as

$$
L(e)=\left\{\begin{array}{l}
0, \text { for }|e| \leq \varepsilon \\
|e|-\varepsilon, \text { otherwise }
\end{array}\right.
$$

It was mentioned in [16] that the solution of the above problem can be formulated in terms of support vectors, $\vec{\theta}=\sum_{i=1}^{N} \beta_{i} \Phi\left(\overrightarrow{x_{i}}\right)$ and the function $\mathrm{f}$ is then written as: 


$$
f(\vec{x}, \vec{\theta})=\sum_{i=1}^{N} \beta_{i}\left\langle\Phi\left(\overrightarrow{x_{i}}\right), \Phi(\vec{x})\right\rangle+b
$$

In (1.5), the inner product $\left\langle\Phi\left(\overrightarrow{x_{i}}\right), \Phi(\vec{x})\right\rangle$ in the feature space is considered as kernel function $K\left(\overrightarrow{x_{i}}, \vec{x}\right)$ [18]. The choice of the kernel function is usually left for users. The kernel function chosen in our work is Gaussian and is defined as:

$$
K\left(\vec{x}, \overrightarrow{x_{i}}\right)=\exp \left[\frac{-\left\|\vec{x}-\overrightarrow{x_{i}}\right\|^{2}}{2 \tau^{2}}\right]
$$

Where $\tau$ is a constant. The coefficients $\beta_{i}$ in (1.5) can be solved by quadratic programming methods with suitable transformation of the above problem into constraint optimization problems and properly rearranging the equation into a matrix form [19],[20] .

According to SVM theory, Support vector regression has the advantage of self-determining its structure. Therefore, there are no initialization problems for SVR. For training data with certain noise distributions the $\varepsilon$-insensitive function [21]. Nevertheless, the robust effects against training data sets with outliers are not obvious in SVR. In this study, we use informal box plots to pinpoint the outlying points in the current training data.

\section{RESULTS}

\section{A. Data preprocessing}

Before applying the regression technique, input data need to be prepared. One essential task is to eliminate outliers. Training data without awareness may lead to unwanted data and may jeopardize function approximation. A close examination of the $1 \mathrm{D}$ plot shows that observations with large errors are inconsistent with the majority of other observations.

Thus, we need a way to detect these observations and deflate their influence. To this end, we use informal box plots [22] to pinpoint the outlying observations. Taking advantage we eliminate around 700 observations. The distance error distribution without outliers is plotted as frequency histogram. Some of the representative statistics that describe much of this distribution error are given in Table1.

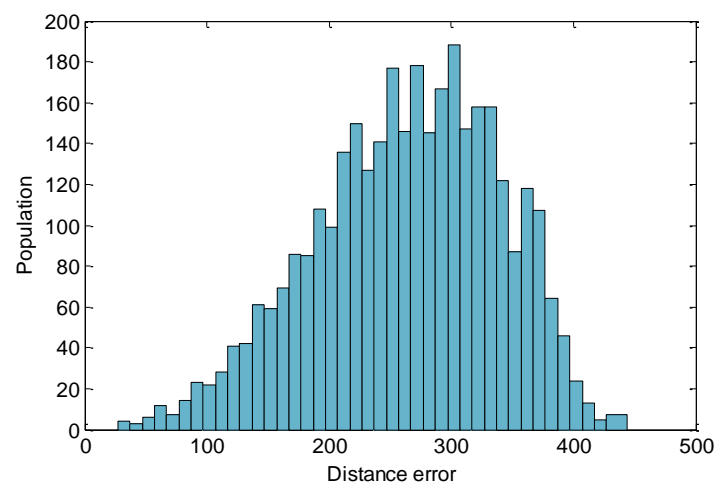

Fig. 5. Distance errors represented as a frequency histogram
TABLE I. ERROR STATISTICS FOR UNCALIBRATED SCALABLE-SPIDAR

\begin{tabular}{|l|l|}
\hline Position error & $\begin{array}{l}\text { Scalable- } \\
\text { SPIDAR }\end{array}$ \\
\hline Mean (mm) & 263.7240 \\
\hline Standard derivation (mm) & 75.6207 \\
\hline Maximum (mm) & 444.2114 \\
\hline
\end{tabular}

The other data preprocessing consideration is scaling. The task of training of regression algorithm is significantly simplified if data lie within a small range. We scale all inputs to have mean zero and standard deviation one.

$$
\left\{\begin{array}{l}
N_{S_{-} S P I D A R}=\left(\left(P_{S_{-} S P I D A R}-\Gamma_{S_{-} S P I D A R}\right) \cdot / \sum_{S_{-} S P I D A R}\right) \\
N_{\text {OTS }}=\left(\left(P_{\text {OTS }}-\Gamma_{\text {OTS }}\right) \cdot / \sum_{\text {OTS }}\right)
\end{array}\right.
$$

Where

$$
\begin{aligned}
& \Gamma_{\text {S_SPIDAR }}=\gamma_{\text {S_SPIDAR }} . J \text { and } \sum_{\text {S_SPIDAR }}=\sigma_{\text {S_SPIDAR }} . J \\
& \Gamma_{\text {отS }}=\gamma_{\text {оTS }} . \mathrm{J} \text { and } \sum_{\text {OTS }}=\sigma_{\text {OTS }} . \mathrm{J}
\end{aligned}
$$

$\gamma_{\text {S_SPIDAR }}$ : Matrix of means of components of the matrix of positions given by the Scalable-SPIDAR haptic device.

$\gamma_{\text {отS }}$ : Matrix of means of components of the matrix of positions given by the Optical Tracking System.

$\mathrm{P}_{\mathrm{S} \_ \text {SPIDAR }}$ : Positions returned by the Scalable-SPIDAR haptic device.

$\mathrm{P}_{\text {OTS }}$ : Positions returned by the Optical Tracking System

$\mathrm{J}$ : Identity matrix

$\mathrm{N}_{\text {S_SPIDAR }}$ : Standardized positions of the Scalable-SPIDAR haptic device.

$\mathrm{N}_{\text {OTS }}$ : Standardized positions of the Optical Tracking System.

\section{B. Calibration using Support Vector Regression}

In the following section, we applied support vector regression (SVR) for calibrating the Scalable-SPIDAR haptic device. The convergence of support vector machine depends on the selection of a kernel function. Kernel functions projects the data into high dimensional feature space. This work uses the Gaussian kernel to perform mapping between ScalableSPIADR and optical tracking system (OTS) data. In the following, trials Gaussian kernel were applied:

$$
K\left(\vec{x}, \overrightarrow{x_{i}}\right)=\exp \left[\frac{-\left\|\vec{x}-\overrightarrow{x_{i}}\right\|^{2}}{2 \tau^{2}}\right]
$$

Where $\tau$ is a constant.

A search was performed for the most effective capacity parameter $\mathrm{C}$ to improve generalization accuracy of the regression technique. The capacity measures the flexibility or richness of regression functions and gives the protection against over fitting. In our experiments, the capacity was set to values between 30 and 100. Another parameter used in the training of support vector regression is epsilon, which checks 
the insensitivity of the regression. The algorithm assumes that estimations that lie within epsilon distance of their true values are enough accurate. Epsilon was chosen to be equal to 0.05 .

To assess the performances of the support vector regression technique, we plot the distribution of position errors but after calibration using support vector regression method. Figure 6 shows that SVR is quite efficient and exhibits lower errors in the overall workspace.
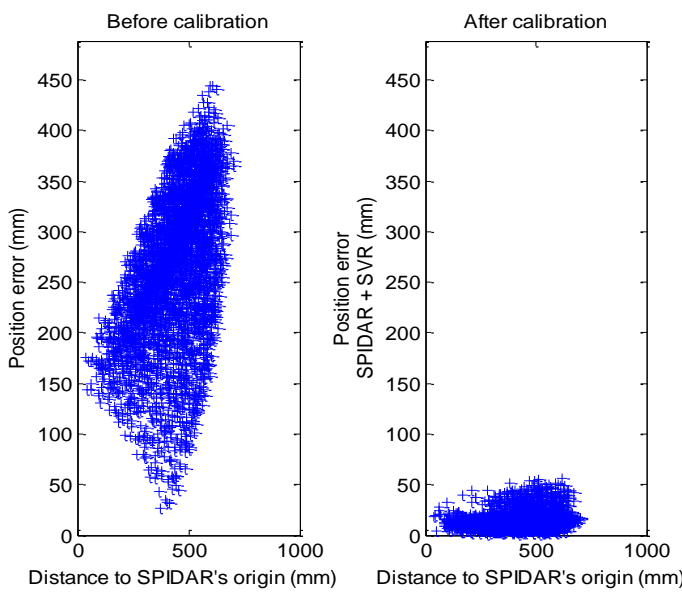

Fig. 6. Distribution of position errors as a function of distance to the Scalable-SPIDAR's origin (before and after SVR calibration)

We can see from the table below that the SVR calibration was successful in reducing the mean error in tracked position form $263.7240 \pm 75.6207 \mathrm{~mm}$ to $12.6045 \pm 8.4169 \mathrm{~mm}$. The maximum possible error is about $57.5928 \mathrm{~mm}$

TABLE II. ERROR STATISTICS BEFORE AND AFTER APPLYING SUPPORT VECTOR REGRESSION TECHNIQUE

\begin{tabular}{|l|l|l|}
\hline Position error & Raw & SVR \\
\hline Mean $(\mathrm{mm})$ & 263.7240 & 12.6045 \\
\hline Standard derivation $(\mathrm{mm})$ & 75.6207 & 8.4169 \\
\hline Maximum (mm) & 444.2114 & 57.5928 \\
\hline
\end{tabular}

After testing support vector regression technique with training data, we need to measure its ability to handle unseen data. The driving idea is to build a testing dataset. To this end, the grip of the Scalable-SPIDAR haptic device was moved in random trajectories of sequential points. These paths represent groups of data points within our predetermined working space. Following this, 512 corresponding point measurements of both the Scalable-SPIDAR and the IR optical tracker can be obtained, therefore allowing evaluating the generalization capability of our technique.

TABLE III. ERROR STATISTICS IN GENERALIZATION

\begin{tabular}{|l|l|l|}
\hline Position error & Raw & SVR \\
\hline Mean (mm) & 267.2019 & 11.6649 \\
\hline Standard derivation $(\mathrm{mm})$ & 75.5131 & 3.8349 \\
\hline Maximum (mm) & 436.1809 & 22.1889 \\
\hline
\end{tabular}

Table 3 shows that SVR keeps convenient performance when handling unseen data. The mean and standard derivation error position values guaranteed by the SVR are good

\section{HYBRID TRACKING SYSTEM}

Due to obstructions of line-of-sight, tracking data reported by the IR optical tracking could be incorrect which leads to a loss of tracking. As a sequence, the consistency between physical and virtual environments is affected, which is revealed by breaks in presence. To overcome this problem, we combine IR optical tracking system (OTS) with a human scale haptic device. The main goal is to maintain an interrupted tracking. In the following section, we provide details on our hybrid system.

Fig 7 illustrates of the pipeline of our hybrid system.

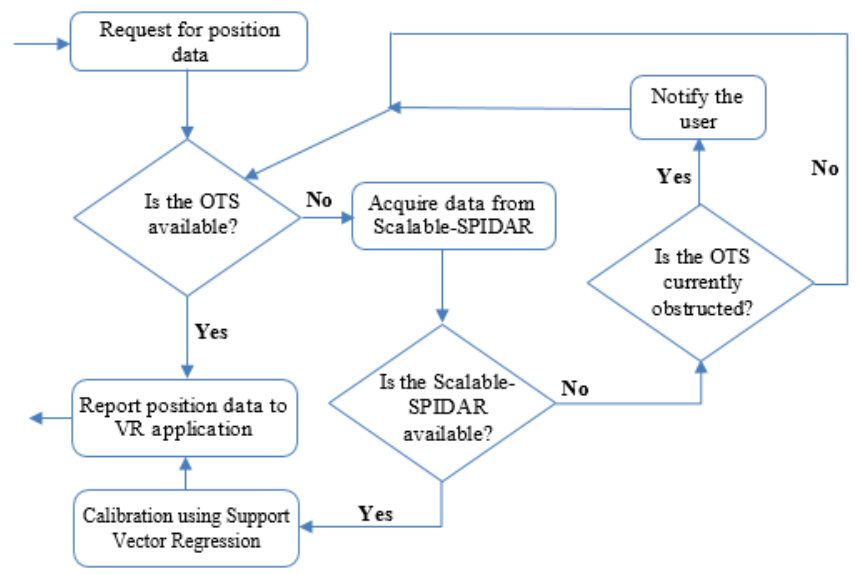

Fig 7. A flow diagram of the hybrid system

Once a request is being sent from the virtual reality application, the system checks whether it can provide data from the OTS. If this optical component loses track then the hybrid system requests position from the haptic component. In this case, the system checks the validity of data and calibrates the Scalable-SPIDAR using the Support vector regression technique. Switching between the two components depends on the optical tracking activity.

\section{CONCLUSION}

In this paper, we have presented a hybrid tracking system for virtual reality applications. The use of support vector regression technique allows for compensation for nonlinear errors in Scalable-SPIDAR position readings. The results show that is possible to reduce the average error between the expected true position and the calibrated position from $263.7240 \pm 75.6207 \mathrm{~mm}$ to $12.6045 \pm 8.4169 \mathrm{~mm}$

These results encourage the development of a hybrid optical- large-scale haptic system for virtual reality applications where the haptic device acts as an auxiliary source of position information for the optical system.

\section{REFERENCES}

[1] G. Welch and E. Foxlin, "Motion tracking: no silver bullet, but a respectable arsenal," Computer Graphics and Applications, IEEE, vol. 22, no. 6. pp. 24-38, 2002. 
[2] M. Frad, H. Maaref, S. Otmane, and A. Mtibaa, "SPIDAR calibration based on regression methods," Networking, Sensing and Control (ICNSC), 2014 IEEE 11th International Conference on. pp. 679-684, 2014.

[3] A. State, G. Hirota, D. T. Chen, W. F. Garrett, and M. A. Livingston, "Superior Augmented Reality Registration by Integrating Landmark Tracking and Magnetic Tracking," in Proceedings of the 23rd Annual Conference on Computer Graphics and Interactive Techniques, 1996, pp. 429-438.

[4] T. Auer and A. Pinz, "Building a hybrid tracking system: integration of optical and magnetic tracking," Augmented Reality, 1999. (IWAR '99) Proceedings. 2nd IEEE and ACM International Workshop on. pp. 1322, 1999.

[5] S. You, U. Neumann, and R. Azuma, "Hybrid inertial and vision tracking for augmented reality registration,” Virtual Reality, 1999. Proceedings., IEEE. pp. 260-267, 1999.

[6] M. Aron, G. Simon, and M. O. Berger, "Handling uncertain sensor data in vision-based camera tracking," Mixed and Augmented Reality, 2004. ISMAR 2004. Third IEEE and ACM International Symposium on. pp. 58-67, 2004.

[7] W. Birkfellner, F. Watzinger, F. Wanschitz, R. Ewers, and H. Bergmann, "Calibration of tracking systems in a surgical environment," IEEE Transactions on Medical Imaging, vol. 17, no. 5. pp. 737-742, 1998.

[8] M. Harders, G. Bianchi, B. Knoerlein, and G. Szekely, "Calibration, Registration, and Synchronization for High Precision Augmented Reality Haptics," Visualization and Computer Graphics, IEEE Transactions on, vol. 15, no. 1. pp. 138-149, 2009.

[9] G. ART Advanced Realtime Tracking, "ARTtrack1 \& DTrack Manual."

[10] L. Buoguila, M. Ishii, and M. Sato, "Multi-Modal Haptic Device For Large-Scale Virtual Environment," ACM Multimed., pp. 277-283, 2000.

[11] Y. Hirata and M. Sato, "3-dimensional Interface Device For Virtual Work Space," Intelligent Robots and Systems, 1992., Proceedings of the 1992 lEEE/RSJ International Conference on, vol. 2. pp. 889-896, 1992.
[12] C.-C. Chuang, S.-F. Su, J.-T. Jeng, and C.-C. Hsiao, "Robust support vector regression networks for function approximation with outliers," IEEE Transactions on Neural Networks, vol. 13, no. 6. pp. 1322-1330, 2002

[13] V. Vapnik, Estimation of Dependences Based on Empirical Data: Springer Series in Statistics (Springer Series in Statistics). Secaucus, NJ, USA: Springer-Verlag New York, Inc., 1982.

[14] R. O. Duda and P. E. Hart, Pattern Classification and Scene Analysis. New Yotk: John Willey \& Sons, 1973.

[15] A. J. Smola, B. Schölkopf, and K.-R. Müller, "The Connection Between Regularization Operators and Support Vector Kernels," Neural Netw., vol. 11, no. 4, pp. 637-649, 1998.

[16] Vladimir N. Vapnik, "The nature of statistical learning theory," IEEE Trans. Neural Netw., 1997.

[17] V. Vapnik, S. E. Golowich, and A. J. Smola, "Support Vector Method for Function Approximation, Regression Estimation and Signal Processing," in Advances in Neural Information Processing Systems 9 -- Proceedings of the 1996 Neural Information Processing Systems Conference (NIPS 1996), 1997, pp. 281-287.

[18] A. Aizerman, E. M. Braverman, and L. I. Rozoner, "Theoretical foundations of the potential function method in pattern recognition learning," Autom. Remote Control, vol. 25, pp. 821-837, 1964.

[19] H. Drucker, C. J. C. Burges, L. Kaufman, A. Smola, and V. Vapnik, "Support vector regression machines," Adv. Neural Inf. Process. Dystems, vol. 1, pp. 155-161, 1997.

[20] a. J. Smola and B. Schölkopf, "On a Kernel-Based Method for Pattern Recognition, Regression, Approximation, and Operator Inversion," Algorithmica, vol. 22, pp. 211-231, 1998.

[21] A. J. Smola, B. Sch, and B. Schölkopf, "A Tutorial on Support Vector Regression,” Stat. Comput., vol. 14, no. 3, pp. 199-222, 2004.

[22] J. W. Tukey, Exploratory Data Analysis. Reading, Mass.: AddisonWesley, 1977. 